\title{
Prospecção Científica e Tecnológica do Uso do Óleo de Coco (Cocos nucifera. $L$ ) na Indústria Alimentícia
}

\section{Scientific and Technological Prospection of the Use of Coconut Oil (Cocos nucifera L.) in the Food Industry}

\author{
Talysie Freire Araújo ${ }^{1}$ \\ Luis Felipe Alves Sousa ${ }^{1}$ \\ Denis Frota Guimarães ${ }^{2}$ \\ Mauro Vinicius Dutra Girão ${ }^{1}$ \\ ${ }^{1}$ Centro Universitário INTA, Sobral, CE, Brasil \\ ${ }^{2}$ Faculdade Alencarina de Sobral, Sobral, CE, Brasil
}

\begin{abstract}
Resumo
O óleo de coco tem se mostrado como um potencial alimento com benefícios nutricionais e funcionais. Este estudo tem como objetivo averiguar o estado da arte, realizando uma prospecção científica e tecnológica em relação ao uso do óleo de coco (Cocos nucifera L.) na indústria de alimentos. Para alcançar os objetivos, foi realizado um levantamento das produções científicas da última década, bem como seu desenvolvimento tecnológico a partir da pesquisa feita em bases de dados. Verificaram-se 1.964 patentes com o óleo de coco, porém apenas três contemplaram os requisitos da pesquisa. Apesar de todos os aspectos positivos que o óleo apresenta, sua utilização para a produção de alimentos na indústria alimentícia se encontra escassa. Diante de todas as evidências apresentadas sobre os benefícios do óleo de coco, sugere-se que pesquisas com esse alimento sejam desenvolvidas a fim de trazer novos estudos que promovam sua inovação tecnológica.
\end{abstract}

Palavras-chave: Nutrição. Alimento Funcional. Inovação.

\begin{abstract}
Coconut oil has become a potential food with nutritional and functional benefits. This study aimed to ascertain the state of knowledge, carrying out a scientific and technological exploration in relation to the use of coconut oil (Cocos nucifera L.) in the food industry. In order to reach the objectives, it was carried out a survey of the scientific productions of the last decade, as well as its technological development based on the research carried out in databases. There were 1964 patents with coconut oil, but only 3 covered the research requirements. Despite all the positive aspects that the oil presents, it is scarce its use for food production in the food industry. In view of all the evidence presented on the benefits of coconut oil, it is suggested that research with this food be developed in order to bring new studies that promote technological innovation for this food.
\end{abstract}

Keywords: Nutrition. Functional Food. Innovation.

Área Técnologica: Propriedade Intelectual. Propriedade Industrial. 


\section{Introdução}

Cocos nucifera é o nome científico do coqueiro, uma planta de origem asiática que chegou ao Brasil em meados de 1553, mais especificamente na Bahia, se adaptou ao clima tropical e aos poucos foi ganhando espaço na costa brasileira e demais regiões do país. Do seu fruto, o coco, derivam-se muitos produtos, como a água de coco, coco fresco, coco desidratado, óleo de coco. Especificamente, o óleo de coco possui constituição química benéfica à saúde que lhe fornece propriedades únicas, tornando seu uso possível em uma ampla gama de alimentos comestíveis (WARWICK et al., 2006; CHE MAN; MANAF, 2006; PRAKRUTHI et al., 2014; GHOSH et al., 2014; KUMAR; KRISHNA, 2015; COSTA; LIMA, 2018; SAMPAIO; FERREIRA; JUIZ, 2019).

O óleo de coco é um alimento tradicional em países tropicais. Estudos com dietas nativas com alto consumo de óleo de coco mostram que a população que o consome geralmente está em boa saúde. O óleo é conhecido por ter efeitos antivirais e antibacterianos e excelentes propriedades curativas. É uma imitação da natureza da gordura do leite materno humano e, portanto, usada em fórmulas infantis (GOPALA et al., 2010).

As propriedades do óleo de coco são únicas, como sabor suave, odor agradável, elevada resistência ao ranço, faixa de temperatura estreita de derretimento, digestibilidade simples $e$ absorvível. Em geral, o ponto de fusão é baixo variando de $23^{\circ}$ a $26^{\circ} \mathrm{C}$. O óleo de coco contém insaturação a um baixo nível, o que o torna estável para a oxidação (CHE MAN; MANAF, 2006).

Além das vantagens técnicas, o óleo de coco possui componentes bioativos com atividade antioxidante protegendo contra o acúmulo de radicais livres que ocasionam o estresse oxidativo. Esse processo pode alterar gravemente as membranas celulares e outras estruturas, induzindo ao desenvolvimento de doenças crônicas e degenerativas. Os antioxidantes são capazes de interromperem os alvos fisiológicos específicos, modulando a defesa antioxidante essencial para a manutenção da saúde. Um fator de grande importância na modulação do estresse oxidativo é a dieta (PHAM-HUY; HE; PHAMHUY, 2008; BARBOSA et al., 2010; OLIVEIRA; BASTOS, 2011; PRAKUTHI et al., 2014).

Os produtores e as indústrias, visando uma melhor qualidade do produto ofertado, buscam novas estratégias para o aumento da produção e aperfeiçoamento da matéria-prima ou produto final. Diversos fatores influenciam esses processos, entre eles os fatores técnicos e ambientais (TOLEDO et al., 2012).

O fruto do coqueiro tem na área alimentícia sua principal aplicação. Acredita-se que o desenvolvimento de tecnologias na área de alimentos proporcione inovação e aperfeiçoamento de técnicas, produtos e processamentos, buscando atender às necessidades alimentares contemporâneas, que encaram dificuldades como superpovoamento, acessibilidade, saúde, desperdício, sustentabilidade, viabilidade econômica, qualidade, entre outras. As diversas aplicações podem se tornar novos produtos, que, para serem protegidos, devem ser patenteados (PASCOAL; SILVA; DRUZIAN, 2013; MUELLER; PERUCCHI, 2014; SAMPAIO; FERREIRA; JUIZ, 2019).

Patente é o direito concedido pelo estado ao inventor titular ou seus legatários à propriedade intelectual sobre uma invenção, um procedimento de fabricação ou aprimoramento de produtos e processos já existentes (INPI, 2017).

A prospecção tecnológica de patentes é a forma de consulta das patentes publicadas. É um importante instrumento para divulgação do conhecimento tecnológico, uma ferramenta indis- 
pensável para a cadeia produtiva do conhecimento, pois fornece um meio para tentar prever quais são os limites e as possíveis novas aplicações de uma dada tecnologia, permitindo à ciência avançar de forma coordenada rumo a um cenário desejado em termos de inovação tecnológica para a indústria, economia e sociedade (QUINTELLA et al., 2011; FREITAS; OLIVEIRA, 2013; BIAGGI et al., 2014; PASCOAL; SILVA; DRUZIAM, 2013).

Empresas, governos $e$ instituições de pesquisas nacionais $e$ internacionais apresentam interesse em tecnologias vinculadas ao uso do fruto do coqueiro como matéria-prima para fabricação de novas tecnologias com aplicações nos setores médico, automotivo, agrícola, têxtil, cosmético e alimentício (TOMMASI et al., 2014; SANTOS; MARTINEZ; JUIZ, 2019; COSTA; LIMA, 2018; SAMPAIO; FERREIRA; JUIZ, 2019).

Atualmente, existe um crescente interesse dos componentes bioativos aliados aos alimentos, por isso, o óleo de coco apresenta-se como um alimento que pode ser utilizado de várias formas, entre elas, na indústria alimentícia. Partindo do presente pressuposto, a pesquisa terá como pergunta norteadora: Quais são as evidências científicas e patenteadas sobre os produtos alimentícios que possuem óleo de coco e quais benefícios nutricionais estão elencados ao seu consumo?

O estímulo à inovação e à pesquisa científica e tecnológica no ambiente produtivoé uma medida que visa à capacitação tecnológica, ao alcance da autonomia tecnológica e ao desenvolvimento do sistema produtivo nacional e regional do país (BRASIL, 2016).

Diante do exposto, realizou-se uma prospecção científica e tecnológica para sistematizar e mapear o desenvolvimento científico e tecnológico em relação ao uso do óleo de coco (Cocos nucifera L.) na indústria de alimentos, a fim de influenciar de forma significativa a comunidade acadêmica, a indústria, a economia e a sociedade.

\section{Metodologia}

Trata-se de uma pesquisa documental com abordagem quantitativa e qualitativa exploratória, descritiva e interpretativa, e, entre elas, a prospecção científica do tipo revisão sistemática e a prospecção tecnológica.

O recrutamento da amostra foi realizado durante o primeiro semestre de 2018, avaliando-se a evolução e as tendências tecnológicas por meio do mapeamento dos depósitos de patentes e do levantamento dos dados científicos. Em todas as bases de dados foram realizadas verificações individuais de cada depósito de patente a fim de compor a amostra objeto de estudo.

A prospecção científica foi desenvolvida em bancos de dados eletrônicos, incluindo-se artigos publicados nos últimos 10 anos, sendo analisados quanto à base indexada, ano de publicação e principais achados, tendo como foco central os trabalhos que descreveram as aplicações do óleo de coco em produtos alimentícios. 
Foram realizadas buscas nas bases de dados: Pubmed, Biblioteca Virtual em Saúde (BVS) e BVS Integralidade, Portal de Periódicos CAPES, e foram utilizados como descritores "cocos nucifera" and "oil" e "cocos nucifera" and "oil" and "food" no campo de pesquisa "título".

A escolha pela utilização de termos em inglês levou em conta que a literatura científica é em sua maioria publicada nesse idioma, aumentando a possibilidade de um maior número de resultados recuperados.

A busca de patentes foi realizada no banco de dados do Instituto Nacional da Propriedade Industrial (INPI) do Brasil, disponível em www.inpi.gov.br. Para a busca, clica-se no link pePI-pesquisa em propriedade industrial, após clica-se no nome Patente e seguidamente na página Pesquisa básica, digitam-se no campo as palavras-chave "cocos nucifera" e "óleo", e após clica-se em pesquisar; e na segunda busca utilizam-se as palavras-chave "cocos nucifera" e "óleo" e "alimento".

Ao mesmo tempo em que na base de dados internacionais, a busca consistirá no European Patent Office (Espacenet), disponível em www.epo.org. Na página inicial, clica-se no link Busca avançada, em seguida, no campo Título ou Resumo, e digitam-se as palavras-chave "cocos nucifera" and "oil",e após clica-se em Pesquisar; e na segunda buscautilizam-se as palavras-chave "cocos nucifera" and "oil" and "food".

No European Patent Office (LATIPAT), disponível em lp.espacenet.com, acessa-se a página inicial e clica-se no link Busca avançada, em seguida, no campo Título ou Resumo e digitam-se as palavras-chave da primeira busca, "cocos nucifera" y "aceite",e após clica-se em Pesquisar; para a segunda busca digitam-se as palavras-chave "cocos nucifera" y "aceite" y "comida.

No United States Patent and Trademark Office (USPTO), disponível em www.uspto.gov.br, na página inicial aciona-se o link Patentes, em seguida o link Pesquisar patentes, depois seleciona-se Busca avançada para busca de registro de patentes, logo após, no campo Consulta, digitam-se as palavras-chave para fazer a primeira busca, "cocos nucifera" and "oil"; e na segunda busca, "cocos nucifera" and "oil" and "food".

E na base World Intellectual Property Organization (WIPO), disponível em www.wipo. int, na página inicial clica-se no link Patentscope, depois no link Acesse o Banco de Dados PATENTSCOPE, na nova janela que abrir seleciona-se o campo ao lado da página de cobertura e digitam-se as palavras-chave para fazer a primeira busca, "cocos nucifera" and "oil", e para a segunda busca, "cocos nucifera" and "oil" and "food".

\section{Resultados e Discussão}

Em relação à prospecção científica, realizou-se uma seleção dos artigos (Figura 1) tendo como quantidade total 1.344 documentos. Os artigos prospectados foram publicados de 2007 até 2018. Após a realização da seleção e descarte daqueles relacionados a temas correlatos, os resultados mostraram que nenhum artigo alcançou os objetivos propostos. 
Figura 1 - Etapas metodológicas para a realização da prospecção científica

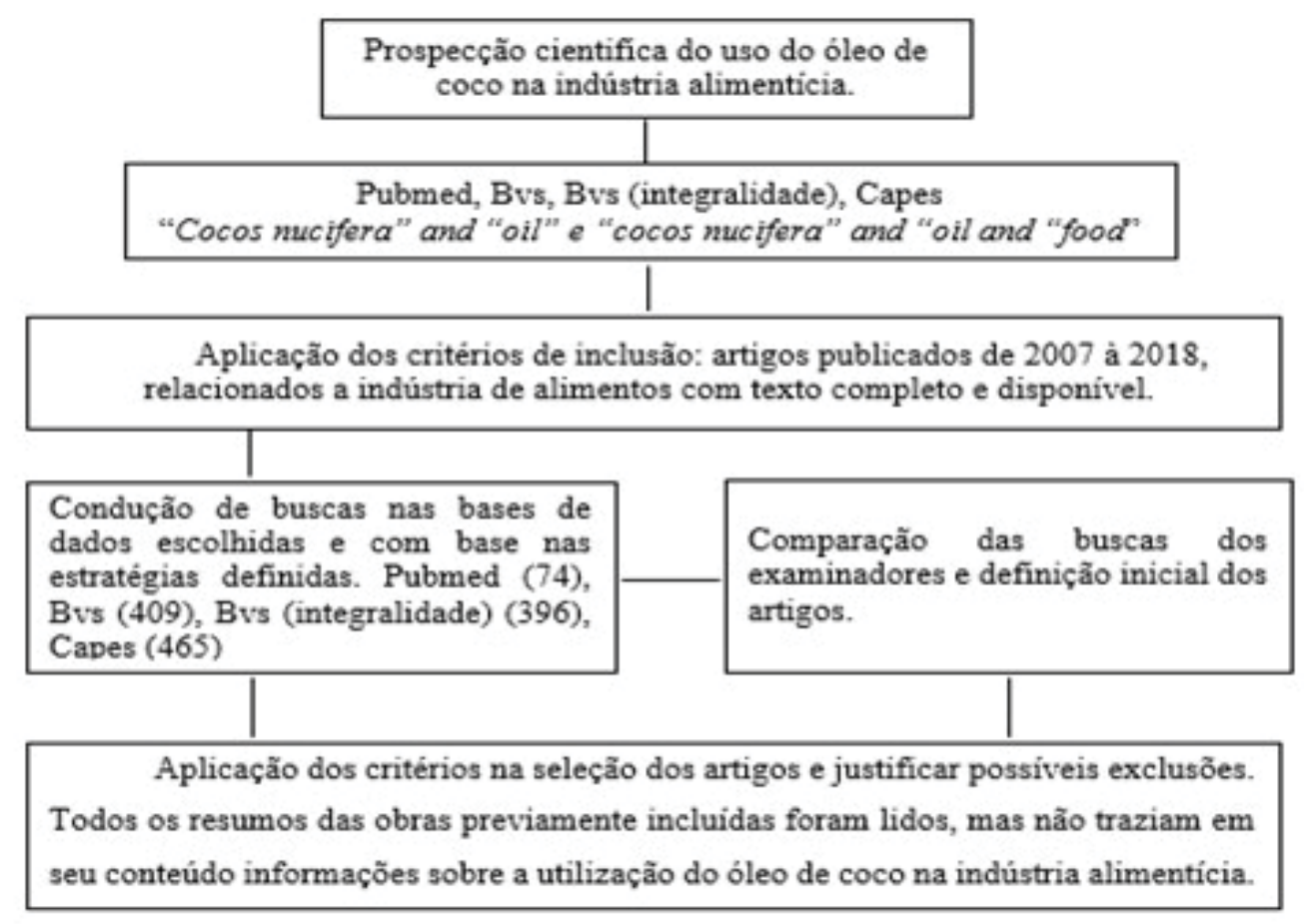

Fonte: Elaborada pelos autores deste artigo (2019)

Após a prospecção efetuada, utilizando-se as combinações "cocos nucifera" and "oil" and "food" e "cocos nucifera" and "oil" (Tabela 1), nenhum artigo foi selecionado, o que demonstra que as pesquisas com o óleo de coco na indústria de alimentos são escassas.

Tabela 1 - Escopo da estratégia da prospecção científica para este estudo

\begin{tabular}{ccccc} 
Palavra-chave & PUBMED & BVS & $\begin{array}{c}\text { BVS } \\
\text { INTEGRAlidade }\end{array}$ & CAPES \\
\hline "cocosnucifera" and "oil" and "food" & 15 & 69 & 65 & 105 \\
\hline "cocos nucifera" and "oil" & 59 & 340 & 331 & 360 \\
\hline
\end{tabular}

Fonte: Elaborada pelos autores deste artigo (2019)

Constatou-se um elevado número de documentos envolvendo o óleo de coco. Entretanto, quando especificado esse termo para o objeto da pesquisa, pode-se observar uma redução significativa de documentos sobre a utilização do óleo de coco na indústria alimentícia.

Estudos com aplicação do óleo de coco na dieta humana continuam a ser desenvolvidos, no entanto, não se tem explorado a aplicação do óleo de coco na indústria de alimentos, o que torna evidente que este tema é um assunto promissor para investimentos de pesquisa, desenvolvimento e apropriação de tecnologia (MAKI et al., 2018; YU; CHO; HWANG, 2018; SILVA et al., 2017).

O mercado potencial para o óleo de coco virgem gradualmente poderá vir a ser reconhecido pelos seus benefícios para a saúde e pelo sabor aplicado ao alimento, conforme apontou um estudo em que $94,1 \%$ dos participantes expressaram reconhecimento pelos alimentos preparados com óleo de coco virgem (PEREZ et al., 2015). 
Em relação à prospecção tecnológica, realizou-se uma seleção das patentes, tendo como quantidade total 1.964 documentos. A pesquisa encontrou patentes relacionadas aos termos utilizados, como mostra a Tabela 2.

A partir da análise do resultado das palavras-chave e suas combinações, foi avaliado o número de pedidos de patentes por base estudada. Embora constem 1.964 documentos nas bases pesquisadas sobre as patentes relacionadas a temas correlatos, verificou-se que alguns foram publicados em mais de uma base, permitindo assim considerar a existência de apenas três patentes que alcançaram os objetivos propostos. As patentes prospectadas foram depositadas em 2015 e 2016. Esses resultados evidenciam a limitação de produtos sobre o tema.

Em relação ao depósito em mais de uma base, alguns países são escolhidos como primeira opção no momento do depósito, e de acordo com a estratégia adotada pelos depositantes, a mesma invenção pode ser depositada em outros países (TOMMASI et al., 2014).

O primeiro pedido de patente ocorreu em 2015 e seu objetivo refere-se a alimento do tipo sorvete utilizando cetella indiana em sua composição. O sorvete formulado, aromatizado com folhas de Hydrocotyle indiano moído (Centella asiática), foi preparado de acordo com os seguintes passos: colocar leite condensado escaldante, creme para todos os fins e açúcar juntos; depois colocar óleo de coco virgem (Cocos nucifera) e solo indiano Hydrocotyle (Cocos asiática), misturar muito bem e colocar em um recipiente de aço inoxidável; em seguida, congelar toda a mistura.

O segundo pedido de patente ocorreu em 2016 e seu objetivo tecnológico refere-se a alimento do tipo biscoito utilizando farinha de soja e farinha de trigo em sua composição e também óleo de coco. O presente modelo de utilidade é um biscoito de soja (Glycinemax) e óleo de coco (Cocos nucifera) incluindo o seu processo de produção. A composição dos biscoitos consiste em 24,68g de farinha de soja, 30,13g de farinha de trigo, 3,98g de óleo de coco, 39,81g leite fres$\mathrm{co}, 0,8 \mathrm{~g}$ de sal, $0,53 \mathrm{~g}$ de levedura e $0,07 \mathrm{~g}$ de bicarbonato de sódio. O produto contém $23,34 \mathrm{~g}$ proteína e 47,68g carboidratos. O biscoito desse modelo de utilidade tem alto teor de proteína e alto teor energético e pode ajudar a diminuir a desnutrição proteico-energética no país.

Tabela 2 - Estratégia da prospecção tecnológica utilizando-se descritores em cada base de dados

\begin{tabular}{|c|c|c|c|c|c|c|}
\hline PalaVRa-CHAVE & EPO & LATIPAT & INPI & JPO & WIPO & USPTO \\
\hline "cocos nucifera" y "aceite" & - & 0 & - & - & - & - \\
\hline "cocos nucifera" y "aceite" y "comida" & - & 0 & - & - & - & - \\
\hline "cocos nucifera" e "óleo" & - & - & 2 & - & - & - \\
\hline "cocos nucifera" e "óleo" e "alimento" & - & - & 0 & - & - & - \\
\hline "cocosnucifera" and "oil" & 29 & - & - & 0 & 46 & 1121 \\
\hline "cocosnucifera" and "oil" and "food" & 0 & - & - & 0 & 2 & 764 \\
\hline
\end{tabular}

Fonte: Elaborada pelos autores deste artigo (2019)

Observa-se que existe um reduzido número de publicações que envolvem o tema em pauta, indicando que produtos alimentícios elaborados a partir desse óleo podem vir a ser bastante inovadores.

A prospecção tecnológica é um estudo centrado nas mudanças tecnológicas, essencial no mapeamento do desenvolvimento científico e tecnológico do país, podendo influenciar a indústria, a economia ou a sociedade de forma significativa. Em diversos países, o método é 
utilizado tanto na orientação de desenvolvimento e inovação das pesquisas como na prospecção acadêmica e científica (SOUSA; OLIVEIRA; CHAVES, 2016).

Além disso, a prospecção tecnológica é um instrumento de base para os gestores na formulação do plano estratégico da organização e na decisão quanto à aplicação de recursos para áreas de pesquisa, ciência e tecnologia. Funciona como um instrumento de apoio ao processo decisório, tendo em vista a possibilidade de se ter uma visão organizada sobre as dinâmicas da pesquisa, desenvolvimento e inovação de um setor e de tecnologias, em curto espaço de tempo. Destaca-se também a potencialidade da informação estratégica que as patentes podem oferecer no sentido de amparar um processo de gestão e monitoramento tecnológico (AMPARO; RIBEIRO; GUARIEIRO, 2012; TEIXEIRA et al., 2013).

A pesquisa nas bases nacionais e internacionais, quando não limita-se à aplicação do óleo de coco a uma área específica, revela que há um número significante de patentes depositadas. Esse número se reduz significativamente quando o foco se destina à produção de alimentos. O Brasil apresentou dois documentos envolvendo óleo de coco, mas estes não foram incluídos por não contemplarem o escopo do estudo.

Mesmo o Brasil sendo um dos maiores produtores do fruto e também possuir uma área geográfica adequada e com condições climáticas favoráveis, o número de depósitos de produtos e processos tecnológicos realizados no país ainda é reduzido (SANTOS; MARTINEZ; JUIZ, 2019; SAMPAIO; FERREIRA; JUIZ, 2019).

Sabe-se que o Brasil tem possibilidade de avançar em P\&D para invenções patenteáveis entre a pesquisa pública e a privada, abrindo possibilidades para a oferta de tecnologias ainda mais aplicáveis a escalas comerciais e não apenas laboratoriais, e assim, alcançar importantes benefícios econômicos e sociais (TOMMASI et al., 2014; SANTOS; MARTINEZ; JUIZ, 2019).

De acordo com a técnica utilizada para a extração da gordura, o óleo pode ter diferentes qualidades, tornando-se possível sua utilização em uma ampla gama de alimentos comestíveis como uma importante fonte de energia, e pode ser incluído para cozinhar, ou como óleo de fritura, encurtamento, margarina e para confeitaria e padaria. Devido à sua palatabilidade, pode ser usado como um substituto para o soro de leite coalhado, queijo e sorvete (CHE MAN; MANAF, 2006; GHOSH et al., 2014; DUMANCAS et al., 2016).

A prospecção tecnológica demonstrou que existe um número escasso de depósitos de patentes relacionadas ao uso do óleo de coco na produção alimentícia, evidenciando o pequeno número de inovações tecnológicas sobre o tema em pauta e que as amplas aplicações vêm sendo subexploradas pela indústria alimentícia mundial.

Diante do número de patentes encontradas, surgea necessidade de um estudo acentuado para o desenvolvimento de pesquisas e inovações com óleo de coco na elaboração de produtos alimentares que tragam benefícios à saúde.

O número de patentes pode refletir o desenvolvimento tecnológico, indicando a necessidade de incentivo nesse campo e na busca do desenvolvimento de outras tecnologias (DIAS et al., 2016).

Em relação à classificação internacional de patentes e ao objetivo tecnológico, as patentes incluídas nesse estudo são classificadas como A21D13/00, A21D2/36, A23G9/00; A23G9/04. A CIP é um modelo valoroso na otimização das buscas nas bases de patentes em que estas 
recebem classificação de acordo com sua aplicação. A CIP está dividida em 8 seções, 21 subseções, 120 classes, 628 subclasses e 69.000 grupos (SOUSA; OLIVEIRA; CHAVES, 2016).

Com base na CIP, os registros de patentes relacionados ao "cocos nucifera" and "oil" e "cocos nucifera" and "oil" and "food" foram distribuídos em uma seção de aplicação: seção "A" contendo dois registros de classificação nas subclasses: A21D, que se refere a aplicações no tratamento, preservação de farinha ou massa, adição de materiais; cozimento; produtos de padaria; preservação do mesmo; e A23G, que se refere ao cacau; produtos de cacau, chocolate; substitutos para produtos de cacau ou cacau; goma de mastigação; gelado; preparação do produto.

O óleo é conhecido por ter efeitos antivirais e antibacterianos e excelentes propriedades curativas. É uma imitação da natureza da gordura do leite materno humano e, portanto, usada em fórmulas infantis. Devido a esses e a outros atributos, a indústria alimentícia tem mostrado interesse por esses compostos que podem agregar maior valor aos produtos (SILVA et al., 2010; RACETTE et al., 2015; PRAKRUTHI et al., 2014; GOPALA et al., 2010). Mesmo com essas potencialidades, não foram encontrados depósitos nessa área.

Em relação ao número de patentes por país de origem, Filipinas é o único país que detém as patentes acerca do tema. Percebe-se que os inventores são apenas uma universidade e uma escola profissionalizante.

Isso pode ser justificado pelo fato de a maior parte da área plantada com coqueiro no mundo situar-se na Ásia. A produção mundial de coco é fortemente influenciada na Índia, Sri Lanka, Filipinas e Indonésia, contribuindo com $76,61 \%$, o que evidencia o coco como uma matéria-prima de grande importância. Esses quatro países juntos detêm cerca de 74,91\% da área total sob plantio de coco e contribuem com $73,61 \%$ para a produção mundial do fruto. A comercialização dos produtos derivados se concentra em grande parte no óleo de coco. As Filipinas destacam-se como a maior área cultivada, sendo o segundo maior país produtor do mundo e o maior exportador do óleo de coco, que corresponde a 7\% do total de suas exportações (GOPALA et al., 2010; SANTOS et al., 2013; MARTINS; JESUS JÚNIOR, 2011; FAO, 2018).

O coco é uma das culturas agrícolas mais importantes na região Ásia-Pacífico e a maioria das plantações é de pequenas propriedades. Nas Filipinas, com apoio financeiro internacional os pequenos produtores adotam o cultivo como fonte alternativa de subsistência tendo acesso a sementes certificadas, equipamentos para o plantio, variedades de culturas melhoradas e novos métodos de agroecologia (FAO, 2017).

Existe um interesse mundial por produção de tecnologias a partir de recursos naturais. O Brasil, um país dotado de uma das mais diversificadas biodiversidades mundiais, torna-se, portanto, um potencial desenvolvedor de invenções patenteáveis (SANTOS; MARTINEZ; JUIZ, 2019; SAMPAIO; FERREIRA; JUIZ, 2019).

Concorda-se com Teixeira et al. (2013) sobre o grande potencial químico e alimentício dos produtos agrícolas relacionados a cocos; dessa forma, são necessárias e promissoras as pesquisas em relação a tais produtos.

Devido ao reduzido número de depósitos de patente brasileiro sobre as aplicações tecnológicas da Cocos nucifera, torna-se necessário um melhor investimento em Pesquisa e Desenvolvimento de novas tecnologias por parte dos centros de pesquisas, universidades e instituições de fomento para o estímulo de se criar invenções patenteáveis correlacionados com o referido 
tema. Tendo em vista o fato de que pesquisas demandam tempo e recursos para mostrarem os primeiros resultados, ressalta-se a necessidade de investimento na área quanto antes, a fim de gerar lucros para o Brasil (TOMMASI et al., 2014; SAMPAIO; FERREIRA; JUIZ, 2019).

Nota-se que o Brasil não possui representantes entre os que mais depositam pedidos de patentes relacionados a aplicações tecnológicas usando coco, apesar de possuir uma excelente disponibilidade de matéria-prima e centros de pesquisas, universidades e empresas atuando no setor. Mesmo com essas potencialidades, ainda há um grande desafio, visto que diversas variáveis, como criar o longo ciclo da cultura, manter uma produção estável, ter as características dos produtores e das propriedades e a baixa tecnificação, devem ser consideradas por estarem relacionadas ao reduzido número de depósitos (TOMMASI et al., 2014).

Analisando a situação atual das patentes e das publicações científicas sobre o óleo de coco na indústria alimentícia, é possível observar um número reduzido de produções. Esse escasso número deve servir de estímulo para os pesquisadores e inventores. Essa matéria-prima atualmente apresenta um grande potencial tecnológico e pode, em um futuro próximo, se tornar uma boa fonte de pesquisa, desenvolvimento e inovação para a indústria alimentícia.

\section{Considerações Finais}

Diante do exposto, torna-se evidente a necessidade do melhor aproveitamento do óleo de coco como recurso na indústria alimentícia e mais investimentos para pesquisa, resultando em conhecimento científico, desenvolvimento de produtos e benefícios econômicos para a sociedade.

Os alimentos utilizando o óleo de coco poderão vir a contribuir para a geração de emprego e renda em toda a cadeia produtiva e trazer benefícios à saúde humana, inclusive nos países produtores de matéria-prima, pois a produção de alimentos com a inclusão do óleo de coco pode integrar inovação para gerar saúde.

Essa prospecção é de pertinência para destacar o estado da arte em pesquisas e produtos envolvendo o óleo de coco, porém o campo ainda é aberto, tanto na área cientifica quanto na área tecnológica, visto que o número de patentes é muito pequeno. Sugere-se, portanto, um maior aproveitamento do potencial nutricional do óleo de coco.

Os resultados aqui apresentados mostram que a aplicação do óleo de coco na indústria alimentícia é um campo promissor ainda em aberto para pesquisa, desenvolvimento e inovação.

\section{Referências}

AMPARO, K. K. S.; RIBEIRO, M. C. O.; GUARIERO, L. L. N. Estudo de caso utilizando mapeamento de prospecção tecnológica como principal ferramenta de busca científica. Revista Perspectivas em Ciência da Informação, [S.l.], v. 17, n. 4, p. 195-209, out.-dez. 2012. Disponível em: http:// portaldeperiodicos.eci.ufmg.br/index.php/pci/article/view/1533. Acesso em: 6 set. 2017.

BARBOSA, K. B. F. et al. Estresse oxidativo: conceito, implicações e fatores modulatórios. Rev.

Nutr., Campinas, v. 23, n. 4, p. 629-643, jul.-ago., 2010. Disponível em: http://www.scielo.br/scielo. php?script=sci_arttext\&pid=S1415-52732010000400013. Acesso em: 18 out. 2017. 
BIAGGI, D. E. et al. Estratégia de difusão da informação tecnológica existente nas bases de patentes como conteúdo educacional para a formação de estudantes do nível técnico e tecnológico. Cadernos de Prospecção, Salvador, v. 7, n. 2, p. 237-246, abr.-jun. 2014. Disponível em: https:// portalseer.ufba.br/index.php/nit/article/view/11352. Acesso em: 20 nov. 2017.

BRASIL. Lei n. 13.243, de 11 de janeiro de 2016. Diário Oficial da República Federativa do Brasil. Brasília, DF, 14 jan. 2016. Disponível em: http:/www.planalto.gov.br/ccivil_03/_ato20152018/2016/lei/l13243.htm. Acesso em: 5 set. 2017.

CHE MAN, Y. B.; MANAF, M. A. Medium chain triacylglycerol. In: Shahidi, F. (ed.). Nutraceutical and specialty lipids and their co-products. Boca Roton: Taylor \& Francis, 2006. p. 27-46. Disponível em: s1.downloadmienphi.net/file/downloadfile3/206/1396380.pdf. Acesso em: 5 set. 2017.

COSTA, H. K. S.; LIMA; L. C. P. Fibra de coco: estudo exploratório sobre registro de patentes. Cadernos de Prospecção, Salvador, v. 11, Edição Especial, p. 387-398, abr.-jun. 2018. Disponível em: https://portalseer.ufba.br/index.php/nit/article/view/23213/FIBRA\%20DE\%20COCO\%3A\%20 ESTUDO\%20EXPLORAT\%C3\%93RIO\%20SOBRE\%20REGISTRO\%20DE\%20PATENTES. Acesso em: 3 out. 2019.

DIAS, I. C. L. et al. Prospecção científica e tecnológica sobre métodos de detecção de agrotóxicos em amostras de água. Revista GEINTEC, [S.l.], v. 6, n. 1, p. 2.874-2.884, 2016.

DUMANCAS, G. G. et al. Health benefitsofvirgincoconut oil. In: HOLT, B. (ed.). Vegetable Oil: properties, uses, and benefits. [S.l.]: Nova Science Publishers, Inc, 2016. Disponível em: https:// www.researchgate.net/profile/Gerard_Dumancas/publication/299371254_Health_benefits_of_virgin_ coconut_oil/links/56f29a2608ae354d162af4da.pdf. Acesso em: 21 out. 2017.

FAO. [Base de dados - Internet]. Food and Agriculture Organization of the United Nations. World Production. 2018. Disponível em: http://www.fao.org/in-action/restoring-coconut-farmerslivelihoodsin-the-philippines/en/. Acesso em: 3 out. 2018.

FREITAS, J. E. F.; OLIVEIRA, L. G. A importância da prospecção para a orientação da pesquisa científica visando a inovação. Revistas Espacios, [S.l.], v. 34, n. 2, p. 6, 2013. Disponível em:http:// www.revistaespacios.com/a13v34n02/13340206.html. Acesso em: 20 nov. 2017.

GHOSH, P. K. et al. Physicochemical and Phytochemical Analyses of Copra and Oil of Cocos nucifera L. (West Coast Tall Variety). International Journal of Food Science, [S.l.], p. 1-8, 2014. Disponível em: https://www.hindawi.com/journals/ijfs/2014/310852/. Acesso em: 25 ago. 2017.

GOPALA, K. A. G. et al. Coconut oil: chemistry, production and its applications - a review.

Indian Coconut J., [S.1.], n. 73, p. 15-27, 2010. Disponível em: https:/www.researchgate.net/ publication/235780162_Coconut_Oil_Chemistry_Production_and_Its_Applications_-_A_Review. Acesso em: 6 set. 2017.

INPI - INSTITUTO NACIONAL DA PROPRIEDADE INDUSTRIAL. Manual para o depositante de patentes. 2017. Disponível em: http:/www.inpi.gov.br/menu-servicos/patente/arquivos/manual-paraodepositante-de-patentes.pdf. Acesso em: 12 ago. 2017.

KUMAR, P. K. P.; KRISHNA, A.G. G. Physicochemical characteristics of commercial coconut oils produced in India. 2015. Disponível em: http://grasasyaceites.revistas.csic.es/index.php/ grasasyaceites/article/view/1527. Acesso em: 7 set. 2017. 
MAKI, K. C. et al. Corn Oil Lowers Plasma Cholesterol Compared with Coconut Oil in Adults with Above-Desirable Levels of Cholesterol in a Randomized Crossover Trial. The Journal of Nutrition Nutrition and Disease. 2018. Disponível em:https:/www.ncbi.nlm.nih.gov/pubmed/30204921. Acesso em: 20 out. 2018.

MARTINS, C. R.; JESUS JÚNIOR, L. A. Evolução da produção de coco no Brasil e o comércio internacional: panorama 2010. Aracajú: Embrapa Tabuleiros Costeiros, 2011. Disponível em: https:/www.infoteca.cnptia.embrapa.br/bitstream/doc/897921/1/doc164.pdf. Acesso em: 15 set. 2018.

MUELER, S. P. M.; PERUCCHI, V. Universidades e a produção de patentes: tópicos de interesse para o estudioso da informação tecnológica. Perspectivas em Ciência da Informação, [S.l.], v. 19, n. 2, p. 15-36, 2014. Disponível em: http://portalde periódicos.eci.ufmg.br/index.php/pci/article/ view/18. Acesso em: 2 set. 2017.

OLIVEIRA, D. M.; BASTOS, D. H. M. Biodisponibilidade de ácidos fenólicos. Quim. Nova, [S.I.], v. 34, n. 6, p. 1.051-1.056, 2011. Disponível em: http://www.producao.usp.br/bitstream/handle/ BDPI/12831/art_OLIVEIRA_Biodisponibilidade_de_acidos_fenolicos_2011.pdf?sequence $=1$. Acesso em: 18 out. 2017.

PASCOAL, D. R. C.; SILVA, I. R. C.; DRUZIAN, J. I. Prospecção tecnológica envolvida no processamento de farinha de banana verde, através de depósitos registrados como propriedade intelectual. In: IV SIMPOSIO INTERNACIONAL DE INOVAÇÃO TECNOLOGICA, 4, Aracajú, SE 25 a 27/09/2013.p. 787-795. Anais [...], Aracajú, SE, 2013. Disponível em: http://www.portalmites. com.br/anaissimtec/index.php/simtec/article/view/80. Acesso em: 2 set. 2017.

PHAM-HUY, L. A.; HE, H.; PHAM-HUY, C. Free Radicals, Antioxidants in Disease and Health. International journal of Biomedical Science, [S.l.], v. 4, n. 2, jun. 2008. Disponível em: https:// www.ncbi.nlm.nih.gov/pmc/articles/PMC3614697/. Acesso em: 20 out. 2017.

PRAKRUTHI, A. et al. Composition of Coconut Testa, Coconut Kernel and its Oil. Journal of the American Oil Chemists Society, [S.l.], v. 91, 2014. Disponível em: https://sci-hub.cc/10.1007/ s11746-014-2447-9. Acesso em: 6 nov. 2017.

QUINTELLA, C. M. et al. Prospecção Tecnológica como uma Ferramenta Aplicada em Ciência e Tecnologia para se Chegar à Inovação. [2011]. Disponível em: http://rvq.sbq.org.br/imagebank/ pdf/v3n5 a05.pdf. Acesso em: 20 nov. 2017.

RACETTE, S. B. et al. Natural Dietary Phytosterols. Journal of International, [S.l.], v. 98, n. 3, 2015. Disponível em: https://www.ncbi.nlm.nih.gov/pubmed/26086252. Acesso em: 21 out. 2017.

SAMPAIO, I.; FERREIRA, S.; JUIZ, P.Estudo Prospectivo Relativo ao Uso do Fruto Coco como Matéria-prima para Fabricação de Cosméticos. Cadernos de Prospecção, Salvador, v. 12, n. 2, p. 314-326, jun. 2019. Disponível em: https://portalseer.ufba.br/index.php/nit/article/ view/27238/17046. Acesso em: 3 out. 2019.

SANTOS, J. R. M. et al. Caracterização físico-química do óleo de coco obtido artesanalmente. [2013]. Disponível em: http://annq.org/eventos/upload/1362501330.pdf. Acesso em: 17 abr. 2017.

SANTOS, D. E.; MARTINEZ; F. C. C.; JUIZ, P. J. L. A Fibra de Coco como Matéria-Prima para o Desenvolvimento de Produtos: uma prospecção tecnológica em bancos de patentes. Cadernos de Prospecção, Salvador, v. 12, n. 1, p. 153-164, mar. 2019. Disponível em: https://portalseer.ufba.br/ index.php/nit/article/view/27230/16969. Acesso em: 3 out. 2019. 
SILVA, M. L. C. et al. Compostos fenólicos, carotenóides e atividade antioxidante em produtos vegetais. Semina: Ciências Agrárias, Londrina, v. 31, n. 3, p. 669-682, jul.-set. 2010. Disponível em: http://www.redalyc.org/pdf/4457/445744097017.pdf. Acesso em: 11 out. 2017.

SOUSA. P.; OLIVEIRA. J. S. S. M.; CHAVES, M. H. Prospecção científica e tecnológica do gênero Simaba Aubl. (Simaroubaceae). Revista GEINTEC, São Cristóvão, SE, v. 6, n. 3, p. 3343-335, 2016. Disponível em: http://www.revistageintec.net/index.php/revista/article/view/852. Acesso em: 12 nov. 2018.

TEIXEIRA, P. R. S. et al. Biossensor do Mesocarpo do Babaçu (Orbignya phalerata) para Detecção de Neurotransmissor: uma Prospecção Tecnológica. Revista GEINTEC, [S.l.], v. 3, n. 3, p. 73-82, 2013. Disponível em: http://www.revistageintec.net/index.php/revista/article/view/131. Acesso em: 12 nov. 2018.

TOLEDO, V. A. A. et al. Produção de geleia real em abelhas africanizadas considerando diferentes ácidos graxos poliinsaturados e fatores ambientais. Gl. Sci. Technol., Rio Verde, v. 5, n. 2, p. 164175, maio-ago. 2012. Disponível em: file://C:/Users/Luiz\%20Junior/Desktop/art\%20coco_abelha. pdf. Acesso em: 20 nov. 2018.

TOMMASI, A. C. et al. Prospecção tecnológica sobre a utilização de óleo de coco para produção de biodiesel. Cadernos de Prospecção, Salvador, v. 7, n. 1, p. 107-116, jan.-mar. 2014. Disponível em: https://portalseer.ufba.br/index.php/nit/article/view/11504/8311. Acesso em: 3 out. 2019.

WARWICK, D. R. N. et al. Coleção plantar. A cultura do coco. 1. ed. Brasília, DF: Embrapa Informação Tecnológica, 2006. p. 9-96. Disponível em: https://www.infoteca.cnptia.embrapa.br/ bitstream/doc/122591/1/00078970.pdf. Acesso em: 26 ago. 2017.

YU. K. S.; CHO. H.; HWANG. K. T. Physicochemical properties and oxidative stability of frying oils during repeated frying of potato chips. Food Sci Biotechnol, [S.1.], v. 27, n. 3, p. 651-659, 2018. Disponível em: https:/www.ncbi.nlm.nih.gov/pmc/articles/PMC6049663/pdf/10068_2017_ Article_292.pdf. Acesso em: 21 maio 2020.

\section{Sobre os Autores}

\section{Talysie Freire Araújo}

E-mail: a.talysie@gmail.com

Nutricionista, Bacharel em Nutrição pelo Centro Universitário Inta (UNINTA). Pós-graduanda em Nutrição Clínica e Funcional pelo Instituto de Pesquisa e Gestão em Saúde (IPGS).

Endereço profissional: Centro Universitário Inta (UNINTA), Rua Antônio Rodrigues Magalhães, n. 359, Dom Expedito, Sobral, CE. CEP: 62050-100.

\section{Luis Felipe Alves Sousa}

E-mail: felipe100.lfa@gmail.com

Graduando em Enfermagem pelo Centro Universitário Inta (UNINTA).

Endereço profissional: Centro Universitário Inta (UNINTA), Rua Antônio Rodrigues Magalhães, n. 359, Dom Expedito, Sobral, CE. CEP: 62050-100. 


\section{Denis Frota Guimarães}

E-mail:denis.frota@gmail.com

Fisioterapeuta, Mestre em Biotecnologia pelo Centro Universitário Inta (UNINTA).

Endereço profissional: Faculdade Alencarina, Estrado do Jordão, s/n, KM 02, Sobral, CE. CEP: 62010-970.

\section{Mauro Vinicius Dutra Girão}

E-mail: viniciusgirao@gmail.com

Biólogo, Mestre em Engenharia de Pesca pela Universidade Federal do Ceará (UFC).

Endereço profissional: Centro Universitário Inta (UNINTA), Rua Antônio Rodrigues Magalhães, n. 359, Dom

Expedito, Sobral, CE. CEP: 62050-100. 